\title{
Introduction to the Minitrack Human-Computer Interaction: Informing Design Utilizing Behavioral, Neurophysiological, and Design Science Methods
}

\author{
Christoph Schneider \\ City University of Hong Kong \\ christoph.schneider@cityu.edu.hk
}

\author{
Joseph S. Valacich \\ University of Arizona \\ valacich@arizona.edu
}

\author{
Angelika Dimoka \\ Temple University \\ dimoka@temple.edu
}

As the Human-Computer Interaction (HCI) minitrack continues to grow we have witnessed new and exciting avenues for exploration. In 2006, this minitrack was developed to provide an outlet for a variety of HCI research streams from a variety of disciplines. In 2013, we began including the disciplines neuroscience and design science, with the aim of Informing Design Utilizing Behavioral, Neurophysiological, and Design Science Methods. Our aim is to get a truly cross-disciplinary understanding of HCI that informs contemporary research and impacts design practices. The papers selected for the competitive HCI minitrack draw on this rich cross-disciplinary tradition. Given that HCI continues to grow and change, we aim to provide a forum for the exchange of novel thoughts and ideas. We believe that the nine papers presented in the three sessions of this minitrack will provide interesting and thought provoking discussions that will be relevant for both research and practitioners.

The papers in the first session present a history of NeuroIS research, as well as methodological advances in HCI research. In the first paper, "The First 10 Years of NeuroIS: A Systematic Literature Review of NeuroIS Publications (2007 - 2017)," Md Rasel Al Mamun, Alsius David, Bin Mai, Dan Kim, and Thomas Parsons analyze and categorize NeuroIS academic research publications. In the next paper, titled "Artifact Sampling: Using Multiple Information Technology Artifacts to Increase Research Rigor," Roman Lukyanenko, Jeffrey Parsons, and Binny Samuel introduce the methodological practice of artifact sampling to address challenges associated with instantiation validity. In the third paper, titled "CLUE: A Usability Evaluation Checklist for Multimodal Video Game Field Studies with Children Who Are Blind," Ticianne Darin, Rossana Andrade, and Jaime Sánchez propose an observational tool for usability field tests involving blind subjects playing video games.

The second session contains three papers related to cognitive aspects of interface design. In the first paper, titled "Visual Aesthetics of E-Commerce Websites: An Eye-Tracking Approach," Ilias Pappas,
Kshitij Sharma, Patrick Mikalef, and Michail Giannakos identify specific patterns of gaze behavior that relate to how users process and perceive visual aesthetics while browsing websites. In the second paper, "Robust User Identification Based on Facial Action Units Unaffected by Users' Emotions," Ricardo Buettner develops a high-performance Random Forests classifier to biometrically identify a user just based on facial actions. In the third paper, "Imaginary Versus Virtual Loci: Evaluating the Memorization Accuracy in a Virtual Memory Palace," Jan-Paul Huttner, David Pfeiffer, and Susanne Robra-Bissantz demonstrate that visualizing loci items in a virtual memory palace can lead to significantly better recall performance.

The final session focuses on novel and innovative interaction technologies. In the first paper, "The Influence of Conversational Agents on Socially Desirable Responding," Ryan Schuetzler, Justin Giboney, Mark Grimes, and Jay Nunamaker examine factors that influence how people disclose sensitive information to automated interviewers. In the second paper, "A Thumb Stroke-Based Virtual Keyboard for Sight-Free Text Entry on Touch-Screen Mobile Phones," Jianwei Lai, Dongsong Zhang, Sen Wang, Isil Kilic, and Lina Zhou present a novel methodology for sight-free text entry on mobile phones. In the final paper, "Interactive Experiences in Public Spaces: A Novel Floor Display System Based on Luminous Modular Units," Jorge Camba, Jeff Feng, and EunSook Kwon describe the design of an interactive concrete floor display system for public spaces that allows for new forms of collaborative interaction in long-term installations.

We would like to sincerely thank the researchers who contributed to this minitrack. Also, we would like to express our thanks for the outstanding efforts put forth by the many reviewers who helped ensure that the papers presented in this minitrack are both interesting and relevant to the HCI field. 